\title{
Ureteral Avulsion During Retrograde Intrarenal Surgery: When the Unexpected Happens
}

\author{
David Denis, MD,, ${ }^{1,2}$ Sergio Moreno, MD, ${ }^{1,3}$ Alfredo Velasco, MD, ${ }^{1,3}$ and José A. Salvadó, MD ${ }^{1,3}$
}

\begin{abstract}
We report the case of a 49-year-old woman affected by bilateral urinary stones. Bilateral semirigid ureteroscopy was performed followed on the left side by a flexible ureteroscopy, caused by localization of the stone. Unfortunately, disinsertion of the left ureter occurred during the intervention. Open conversion was performed in the same single procedure for left ureteral reimplant. After an easy recovery, the patient was rehospitalized on day 9 postintervention for left pyelonephritis. This case report discusses the management of this rare but serious complication, including the necessity for quick thinking and decision making.
\end{abstract}

Keywords: ureteroscopy, flexible ureteroscopy, ureteral avulsion, urolithiasis, ureteral instrumentation

\section{Clinical History}

A 49-YEAR-OLD WOMAN was admitted to our unit for right renal colic. Otherwise healthy, her medical and surgical history is irrelevant, with just one more episode of renal colic 20 years ago with spontaneous stone passage. On admission, she mentioned a typical and invalidating right flank pain that had been bothering her for a period of 6 days. She also reported hematuria, de novo, for a period of 10 days. There had been no fever and she was reporting mild urinary irritative symptoms.

A blood test was performed, dosing a creatinine at $0.99 \mathrm{mg} / \mathrm{dL}$ and a glomerular filtration rate at $59.6 \mathrm{~mL} /(\mathrm{min}$. $1.73 \mathrm{~m}^{2}$ ). There was no hyperleukocytosis, nor electrolyte disorder, and the $\mathrm{C}$-reactive protein was negative. A urine test discarded any urinary infection. CT scan revealed a ureteral stone at the right ureterovesical junction (maximum diameter of $7 \mathrm{~mm} ; 1200 \mathrm{HU}$ ) (Fig. 1), as well as a left renal stone, in the inferior caliceal group (maximum diameter of $6 \mathrm{~mm}$; $980 \mathrm{HU}$ ) (Fig. 2).

After thorough discussion with the patient and because of her preferences, the treatment offered consisted of a singleprocedure bilateral ureteroscopy-the patient was working in the mining industry, far from any emergency department and high-quality health care.

\section{Physical Examination}

Upon admission and before surgery, vital signs were optimal. Physical examination turned out to be strictly normal, with only a slightly painful right renal percussion.

\section{Intervention}

Under general anesthesia, we proceeded to a right retrograde pyelogram, which showed a clear contrast-filling defect in the distal ureter, compatible with the stone described in the CT. After safely ascending two ureteral guidewires, we then performed a right semirigid ureteroscopy. This allowed us to rapidly identify the distal stone, which was easily fragmented with laser and evacuated with forceps. A Double-J stent was then left in place on the right side.

We then started working on the left side. A retrograde pyelogram was performed, here again showing a contrast-filling defect, but this time in the inferior caliceal group. Two ureteral guidewires were installed, followed by a careful semirigid ureteroscopy. The ureteroscope allowed for a proper and careful optical dilatation. An access sheath was then ascended on one of the guidewires, keeping the other one as a safety wire. Everything was done under fluoroscopic guidance. We encountered no real resistance when ascending both the semirigid ureteroscope and the access sheath. This being said, the move was performed by a resident in training, who might not be fully familiar with the level of resistance and thus of force one can apply in such situations. Then, the flexible ureteroscope was introduced in the access sheath and carefully ascended into the kidney. As soon as the ureteroscope got to the superior portion of the access sheath, and out of it, we realized something was wrong. We were obviously not into the left kidney cavities, but most probably into retroperitoneal fat. We took out the flexible ureteroscope and its access sheath, leaving a second guidewire in place - the safety guidewire was never removed - and performed a retrograde

\footnotetext{
${ }^{1}$ Department of Urology, Clinica Santa Maria, Santiago, Chile.

${ }^{2}$ Department of Urology, Cliniques Universitaires Saint-Luc, Bruxelles, Belgique.

${ }^{3}$ Universidad Finis Terrae, Facultad de Medicina, Providencia, Región Metropolitana de Santiago, Chile.
} 


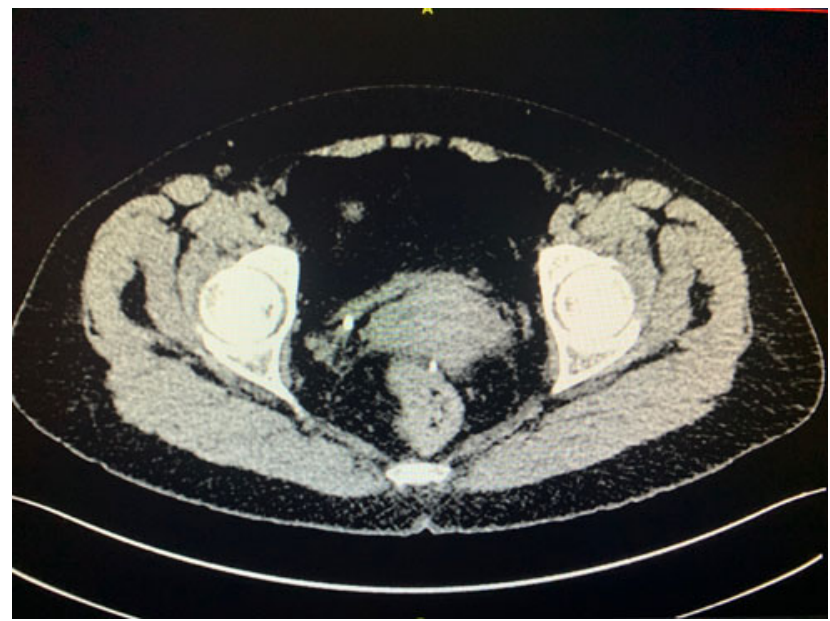

FIG. 1. Ureteral stone at the right ureterovesical junction (maximum diameter of $7 \mathrm{~mm} ; 1200 \mathrm{HU}$ ).

pyelogram (Figs. 3 and 4). We soon realized a clear solution of continuity in the distal left ureter. After analyzing the images for a moment, the diagnosis was quite clear and we opted for open exploration and probably ureteral reimplant. There was a clear and complete solution of continuity in the distal left ureter. There was clearly no room for spontaneous healing with a Double-J stent in place. After obtaining agreement of the family, we converted to open surgery with a left Gibson incision, accessed the retroperitoneum, and finally identified the lesion in the left ureter, which was clearly disinserted in its distal portion. The length of vital ureter was clearly sufficient for a Lich Gregoir reimplant, which was easily performed. A Double-J stent and vesical catheter were installed at the end of the procedure.

\section{Outcome: A Complication Never Comes Alone}

The immediate postoperative evolution was exemplary and the patient was discharged on the fourth postoperative day. Treatment at discharge consisted of analgesics and ciprofloxacine for 7 days. The vesical catheter and right Double-J stent were removed on the eighth postoperative day. On the

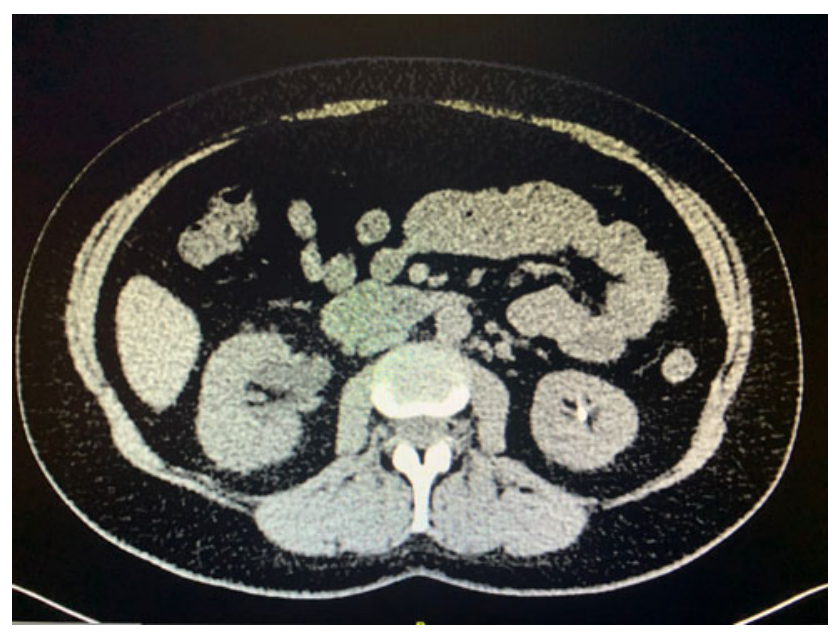

FIG. 2. Left renal stone, in the inferior caliceal group (maximum diameter of $6 \mathrm{~mm} ; 980 \mathrm{HU}$ ).

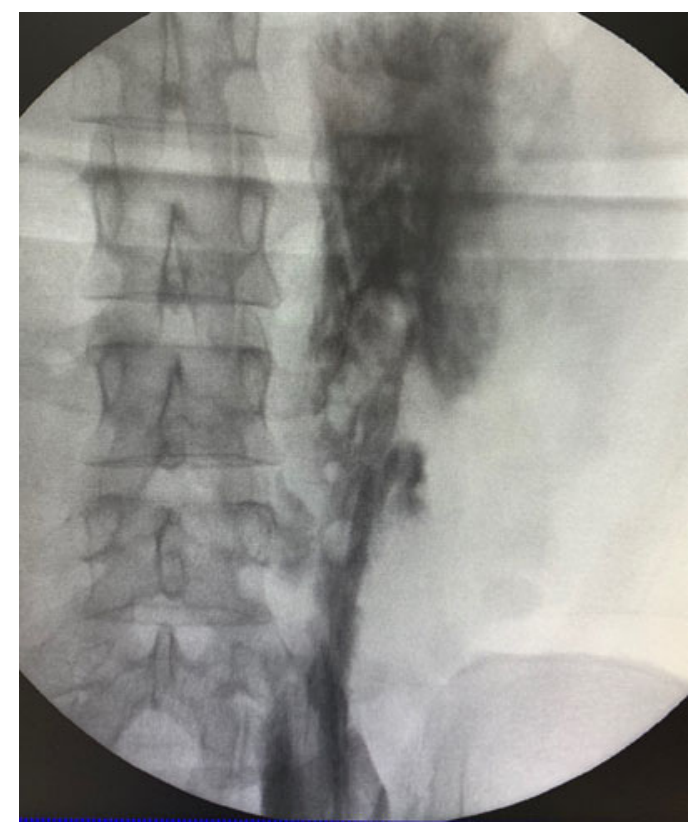

FIG. 3. Solution of continuity in the distal left ureter on retrograde pyelogram.

ninth postoperative day, the patient was readmitted through the emergency department for sepsis of urinary origin. Urine test and culture revealed an infection with $E$. coli (resistant to ciprofloxacine). CT scan showed a well-positioned left Double-J stent, with a slightly dilated left kidney and clear infiltration of the perirenal fat. She was hospitalized for a total of 6 days and received IV antibiotics-originally treated with piperacillin/tazobactam plus amikacin, and then switched to ceftriaxone. She was discharged on the sixth day with oral cefpodoxime for 10 more days. The patient was last seen in

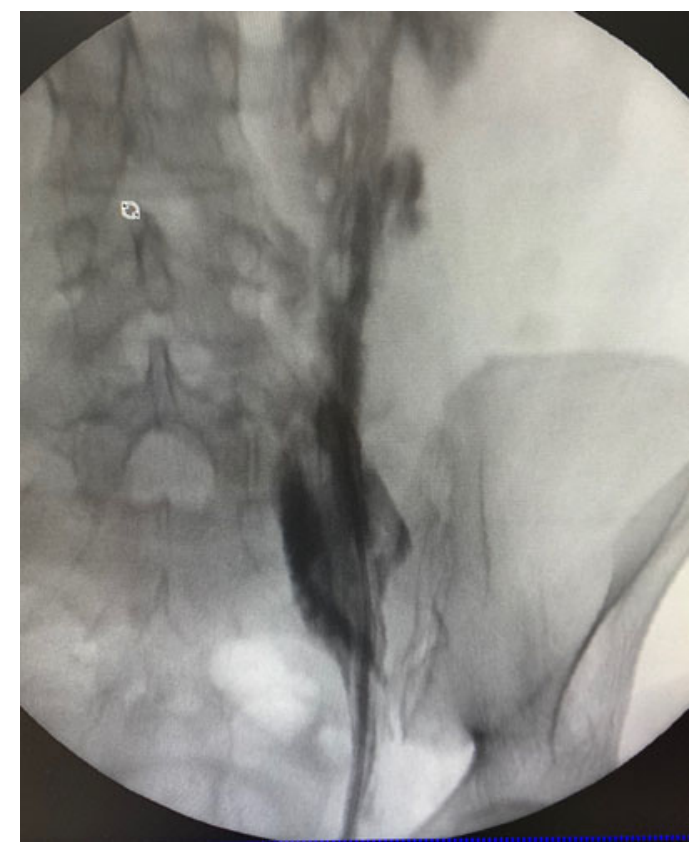

FIG. 4. Solution of continuity in the distal left ureter on retrograde pyelogram. 
control a month later: she had no fever or pain. Urine was sterile and blood test was reassuring, witnessing a neat resolution of inflammatory and infection indicators.

\section{Discussion and Conclusion}

Thanks to a tremendous progress in ureteroscope design, innovations in fiber-optic technology, and accessory instrumentation, ureteroscopy has undergone a dramatic evolution over the past three decades. Although ureteroscopic injury remains the most common cause of ureteral trauma, it is mainly because of the high volume of patients; numerous improvements in the field have resulted in a reduced incidence of serious complications. ${ }^{1}$ The frequency of complications in ureteroscopy varies between $0.5 \%$ and $10 \%$ in the literature, whereas ureteral avulsion-definitely one of the most serious complications, almost always requiring open or laparoscopic surgery-occurs in $0 \%$ to $0.5 \%$ of the procedures. ${ }^{2,3}$ Although ureteral avulsion remains rare, the risk for this serious complication is real and should be kept in mind when performing ureteroscopy. Urologists should be familiar with all avulsion scenarios and the different management options. The paucity of the literature does not help in this regard.

Risk factors have been identified. They include symptomatic stones persisting for $>3$ months, stones with a diameter $>5 \mathrm{~mm}$, stones tightly encapsulated, previous extracorporeal shockwave lithotripsy treatment, etc. ${ }^{3,4}$ Avulsion occurs most commonly during extraction of a basketed stone that is too large to traverse the ureter, which is why the basketing of stones located in the middle and proximal ureter should be attempted with extreme caution only, and always with a safety guidewire in place.

Management options depend on the level and severity of the ureteral lesion. ${ }^{4,5}$ Complete avulsion requires open or laparoscopic repair. If the avulsion involves the distal ureter, ureteroneocystostomy is recommended. Avulsion of the middle third of the ureter may require a psoas hitch or Boari flap. Proximal injuries may be repaired primarily with end-to-end anastomosis over a ureteral stent, provided the ureter has not been devitalized. 5

One question that could arise from this case report, a posteriori, is to wonder why address asymptomatic left renal stones without previous dilatation with Double-J stent. To understand why, the reader should be informed about the particular geography and health care access in Chile. The reality of this particular patient was that she was working in the remote northern part of the country, in the mining industry. She had no easy access to any high-quality urologic care, let alone access to flexible ureteroscopy. Unfortunately, her modest wage was not allowing for multiple trips back and forth, either. This is why, after being fully and duly informed about all the risks, advantages, and potential consequences of such a procedure, she opted for bilateral ureteroscopy during the same single procedure.

In summary, despite significant technological advances, surgical misadventures still occur, some of which have lasting consequences. In our opinion, reporting this case is a first step to raising awareness on those critical iatrogenic situations, calling for a routine reporting of complications and highlighting the need for systematic use of standardized classification systems.

\section{Disclosure Statement}

No competing financial interests exist.

\section{Funding Information}

No funding was received for this article.

\section{References}

1. de la Rosette J, Denstedt J, Geavlete P, et al. The clinical research office of the endourological society ureteroscopy global study: Indications, complications, and outcomes in 11,885 patients. J Endourol 2013;28:131-139.

2. Ibrahim AK. Reporting ureteroscopy complications using the modified Clavien classification system. Urol Ann 2015;7: $53-57$.

3. Somani BK, Giusti G, Sun Y, Osther PJ, Frank M, De Sio M, Turna B, De la Rosette J. Complications associated with ureteroscopy (URS) related to treatment of urolithiasis: The Clinical Office of Endourological Society URS Global Study. World J Urol 2017;35:675-681.

4. Schoenthaler M, Wilhelm K, Kuehhas FE, et al. Postureteroscopic lesion scale: A new management modified organ injury scale-evaluation in 435 ureteroscopic patients. J Endourol 2012;26:1425-1430.

5. Türk C, Skolarikos A, Neisius A, Petř́k A, Seitz K, Donaldson JF, Drake T, Grivas N, Ruhayel Y. EAU guidelines on urolithiasis. Eur Urol 2019

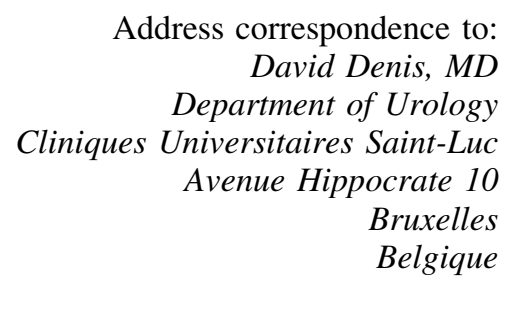

E-mail: david.p.denis@gmail.com

Cite this article as: Denis D, Moreno S, Velasco A, Salvadó JA (2020) Ureteral avulsion during retrograde intrarenal surgery: when the unexpected happens, Journal of Endourology Case Reports 6:3, 177-179, DOI: 10.1089/cren.2020.0006. 